\title{
STABILITY AND BOUNDEDNESS ANALYSIS OF A SYSTEM OF RLC CIRCUIT WITH RESPONSE
}

\author{
A.L. Olutimo ${ }^{1 \S}$, I.D. Omoko ${ }^{2}$ \\ ${ }^{1,2}$ Department of Mathematics \\ Lagos State University \\ Ojo - 23401, NIGERIA
}

\begin{abstract}
This paper presents a stability and boundedness analysis of a system of RLC circuit modeled using a time varying state-space method. Stability problem analysis is very important in RLC circuits. There is some potential for a response characterizing the system of RLC circuit to approach infinity when subjected to certain types of inputs. Unstable circuit causes damage to electrical systems. Analysis of problems of such system stability is carried out using the Lyapunov's theory. In this paper, we provide in simple form, conditions which ensure the stability and boundedness of the state variables $x_{i}(t)$ $(i=1,2)$ characterizing the system of RLC circuit using Lyapunov's second or direct method.
\end{abstract}

AMS Subject Classification: 34D40, 34D20, 34D20, 34C25

Key Words: RLC circuits; state variables; stability and boundedness of system circuit; Lyapunov function

\section{Introduction}

An RLC circuit is a resonant or a tuned circuit consisting of a resistor $(R)$, an inductor $(L)$ and a capacitor $(C)$ connected in series or parallel. An RLC circuit can be described by a linear second order differential equation for circuit analysis. The importance of this is that its characterization is given by its transfer function, which is the frequency domain equivalent of the time domain input-output relation. The knowledge of the interior parameters of the circuit

Received: July 22, 2019

(c) 2020 Academic Publications

$\S$ Correspondence author 


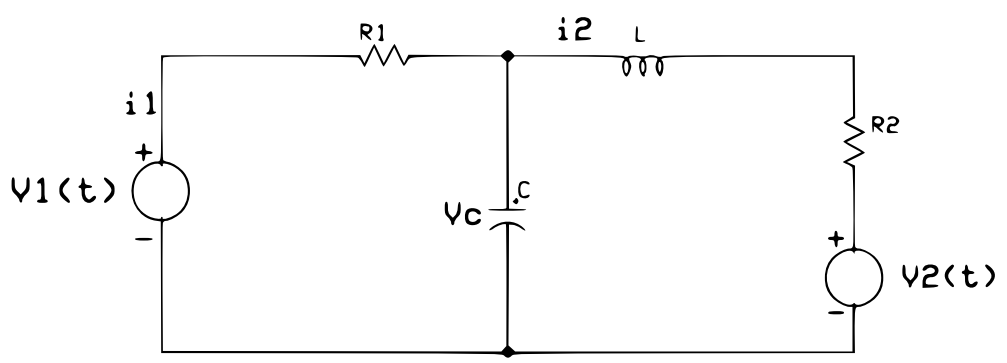

Figure 1: $R L C$ Circuit

is not considered but rather to the control of the closed-loop behavior when feedback control is used. RLC circuit systems are widely applied in electromechanical systems and other technical field of application. The qualitative theory of such system is more complicated to analyze. If we determine input, the state variables and their response vary depending on the circuit. The out response tends to zero as time tends to infinity. If out response of such a system circuit approaches infinity, the circuit is said to be unstable. This undesirable case can be detected theoretically and eliminated at development stage. To address this problem, we make use of Lyapunov theory. Results obtained may be useful to researchers who may want to use it for the development of more general cases like coupled circuits.

The energy storage elements of a system are what make the system dynamic. The flow of energy into and out of a storage element occurs at a finite rate is described by a differential equation relating the derivative of the energy storage variable (a state variable) to other power variable of the element. There are two independent energy storages in RLC circuit, the capacitor which stores energy in an electric field and the inductor which stores energy in a magnetic field. The state variables are the energy storage variables of these two elements, $V_{c}$ and $V_{i L}$. According to Peng and Pileggi [8], state variables are the minimum set of variables that fully describe the system circuit and its response to any given set of inputs. By this, it shows that the qualitative properties of state-determined system like Fig. 1 is completely characterized by the stability and boundedness of the set of $x_{i}(t) \quad(i=1,2)$ variables.

We consider a RLC circuit represented in Fig.1. We suppose also that circuit elements (resistances, inductances and capacitances) are always positive. The first time derivative of the inductance exist. $V_{1}(t)$ and $V_{2}(t)$ are inputs voltages and feed currents $i_{1}, i_{2}$ are finite but undefined. Mathematical equation of the circuit can be different depending on the choice of state variables that are defined. Let the state variables describing the response be $x_{1}(t)=i_{2}(t)$ and 
$x_{2}(t)=V_{c}(t)$. It is also necessary to apply Kirchhoff's laws and by KCL and KVL in Morris [4], we obtain the following system of differential equations:

$$
\begin{aligned}
& \dot{x}_{1}(t)=-\frac{R_{2}}{L} x_{1}(t)+\frac{1}{L} x_{2}(t)-\frac{V_{2}(t)}{L}, \\
& \dot{x}_{2}(t)=-\frac{1}{C} x_{1}(t)-\frac{1}{C R_{1}} x_{2}(t)+\frac{V_{1}(t)}{C R_{1}} .
\end{aligned}
$$

Equation (1) is linear heterogeneous system of two differential equations of the first order which can be represented in matrix notation as

$$
\begin{aligned}
\frac{d}{d t}\left(\begin{array}{c}
x_{1}(t) \\
x_{2}(t)
\end{array}\right) & =\left(\begin{array}{cc}
-\frac{R_{2}}{L} & \frac{1}{L} \\
-\frac{1}{C} & -\frac{1}{C R_{1}}
\end{array}\right)\left(\begin{array}{l}
x_{1}(t) \\
x_{2}(t)
\end{array}\right) \\
& +\left(\begin{array}{cc}
0 & -\frac{1}{L} \\
\frac{1}{C R_{1}} & 0
\end{array}\right)\left(\begin{array}{l}
V_{1}(t) \\
V_{2}(t)
\end{array}\right) .
\end{aligned}
$$

Now, we represent (2) as a linear vector differential equation of order one,

$$
\dot{X}=A X+F(t),
$$

where state vector $X$ is a column vector, $A$ is a $2 \times 2$ matrix of the constant coefficient and $F(t)$ is a free column vector. $A$ is not symmetrical since the circuit is connected through connecting elements. The Lyapunov function approach has been a powerful tool to ascertain the stability of solutions of differential equations as well as the state variables characterizing dynamic systems and an effective method for studying the stability of such systems is still the Lyapunov's second method, see Liapunov [3]. Today, this method is widely recognized as an excellent tool not only in the study of differential equations but also in the theory of control theory systems, dynamical systems, systems with time lag, power system analysis, time varying non-linear feedback systems and so on. However, the construction of these Lyapunov functionals remain a general problem, see Qin et al. [9]. According to Tunc [10] "finding an appropriate Lyapunov function is in general a difficult task". Yao and Meng [11] expressed further: "how do we construct those appropriate Lyapunov functionals? No author has discussed them thus far. It is in general a difficult task". The application of Lyapunov's theory in analysing the behavior of critical variables describing the system of electrical circuits is the lack of straight forward procedure for finding appropriate Lyapunov functionals. However, recently, Olutimo [5] by constructing appropriate Lyapunov functional obtained conditions that ensure the stability of state variables describing the system of DC servo motor 
with load. And very recently, Piriadarshani and Sujitha [7] investigated the stability of feedback control system using transfer function while Lenka [2] established new stability conditions for certain class of nonautonomous fractional order systems using time varying Lyapunov functions. Thus, our problem in Fig. 1 is best treated since it has been reduced to stability problem according to Lyapunov's equations in (1). This Lyapunov's second method lies in constructing a scalar function, $\Phi$ such that it is positive definite and its derivative $\dot{\Phi}$, along the system (1) is negative-semi definite. When these properties of $\Phi$ and $\dot{\Phi}$ are shown to be satisfied according to Lyapunov's theory (Demidovich [1], Yoshizawa [12]), then the behavior of the system circuit is known. The construction of a Lyapunov function $\Phi$, which is a quadratic form satisfying the requirements for $\Phi$ and $\dot{\Phi}$ for discussing the stability and boundedness of the $x_{i}(t)$ variables of the linear vector differential equation $(3)$ for $F(t)=0$ and $F(t) \neq 0$ respectively is obtained. It must be noted here that the physical meaning of Lyapunov function is not considered. In known cases Lyapunov functions were obtained as abstract mathematical approach results, see Pai [6]. Also, in this study we assume that physical meaning availability is not connected with function efficiency.

\section{Stability analysis}

\section{Assumptions:}

In addition to the basic assumption imposed on the elements $R, L$ and $C$ in (1), we also assume that

(i) $\frac{R_{2}^{2}}{L}+\frac{R_{1} R_{2}}{L}+\frac{R_{2}}{C R_{1}}+\frac{1}{C}>0$ and

(ii) $\left|V_{1}(t)-V_{2}(t)\right| \leq \rho(t)$,

where $V_{1}(t)$ and $V_{2}(t)$ are the input and reverse voltages respectively in the circuit.

To establish our result, we employ the following scalar function $\Phi=\Phi\left(x_{1}, x_{2}\right)$ defined by

$$
2 \Phi\left(x_{1}, x_{2}\right)=\left(R_{2}+\frac{L}{C R_{1}}+R_{1}\right) x_{1}^{2}+2 x_{1} x_{2}+\frac{C R_{1}}{L} x_{2}^{2} .
$$

From (4), it is clear that $\Phi(0,0)=0 . \Phi$ in (4) can be arranged as follows,

$$
2 \Phi\left(x_{1}, x_{2}\right)=\left(R_{2}+R_{1}\right) x_{1}^{2}+\frac{L}{C R_{1}}\left(x_{1}+\frac{C R_{1}}{L} x_{2}\right)^{2} .
$$


It is obvious from (5) that the function $\Phi$ defined in (4) is a positive definite function. Hence, there is a positive constant $D_{1}$ such that

$$
\Phi\left(x_{1}, x_{2}\right) \geq D_{1}\left(x_{1}^{2}+x_{2}^{2}\right) .
$$

Now, consider the case of a natural process where dissipation is absent that is, $F(t)=0$ in $(3)$.

On using (4), a direct differentiation of $\frac{d \Phi}{d t}$ along the system (1) gives after simplification of $\Phi$, yields

$$
\dot{\Phi}(t) \leq-\left(\frac{R_{2}^{2}}{L}+\frac{R_{1} R_{2}}{L}+\frac{R_{2}}{C R_{1}}+\frac{1}{C}\right) x_{1}^{2} .
$$

It follows that $\frac{d \Phi\left(x_{1}, x_{2}\right)}{d t}=0$ if and only if $x_{1}=0, \frac{d \Phi\left(x_{1}, x_{2}\right)}{d t}<0$ for $x_{1} \neq 0$ and $\Phi(0,0) \geq 0$.

Thus, the system of RLC circuit in Fig. 1 is stable according to Lyapunov's theory if Assumption (i) is satisfied from the time $t_{o}$ to $t \rightarrow \infty, t \geq t_{o}$. In this case, the impulse response approaches zero as $t \rightarrow \infty$.

\section{Boundedness analysis}

We now consider the case $F(t) \neq 0$ in (3) where dissipation is present due to external conductive connections or elements.

The conclusion of (7) can be revised as follows

$$
\dot{\Phi}(t)=\frac{d \Phi}{d t} \leq k_{1}\left|V_{1}-V_{2}\right| x_{1}+k_{2}\left|V_{1}-V_{2}\right| x_{2},
$$

where $k_{1}=\max \left\{\frac{V_{1}}{C R_{1}}, \frac{R_{2}}{L}+\frac{1}{C R_{1}}+\frac{R_{1}}{L}\right\}$ and $k_{2}=\left\{\frac{1}{L}\right\}$.

By noting Assumption (ii), we have

$$
\dot{\Phi}(t) \leq\left(k_{1}\left|x_{1}\right|+k_{2}\left|x_{2}\right|\right) \rho(t) .
$$

On using inequality $|x| \leq 1+x^{2}$, we get

$$
\dot{\Phi}(t) \leq k_{3}\left(2+x_{1}^{2}+x_{2}^{2}\right) \rho(t),
$$

where $k_{3}=\max \left\{k_{1}, k_{2}\right\}$.

Thus,

$$
\dot{\Phi}(t) \leq 2 k_{3} \rho(t)+k_{3}\left(x_{1}^{2}+x_{2}^{2}\right) \rho(t) .
$$


Since $D_{1}\left(x_{1}^{2}+x_{2}^{2}\right) \leq \Phi\left(x_{1}, x_{2}\right)$, by (6) it follows that

$$
\dot{\Phi}(t) \leq 2 k_{3} \rho(t)+k_{3} D_{1}^{-1} \Phi\left(x_{1}, x_{2}\right) \rho(t) .
$$

We assume that the input is conditioned to be finite and controllable, thus we have that

$$
\left|\int_{0}^{t} \rho(s) d s\right| \leq D_{2}, \quad D_{2}>0 \text { for all } t \geq 0 .
$$

Integrating (8) from 0 to $t$ with the above assumption and Gronwall-ReidBellman inequality, we obtain

$$
\Phi\left(x_{1}, x_{2}\right) \leq\left(\Phi(0,0)+2 k_{3} D_{2}\right) \exp \left(k_{3} D_{1}^{-1} D_{2}\right) .
$$

Now, since the right hand side of $(9)$ is a constant and $\Phi\left(x_{1}, x_{2}\right) \rightarrow \infty$ as $x_{1}^{2}+x_{2}^{2} \rightarrow \infty$, it follows that there exist a positive constant $D_{3}$ such that

$$
\left|x_{1}(t)\right| \leq D_{3} \text { and }\left|x_{2}(t)\right| \leq D_{3} \text { for } t \geq 0 .
$$

Thus, this implies that every bounded input produces a bounded output if the stated assumptions are satisfied.

\section{Stability analysis for system (1)}

The plot of $x_{1}(t), x_{2}(t)$ of equations (1) which are the critical variables characterizing the RLC circuit in Fig. 1. is shown in Fig. 2, Fig. 3 and Fig. 4 below. It is very clear from Fig. 2, Fig. 3 and Fig. 4 that Assumption (i) obtained by the Lyapunov functional (4) is satisfied for all values of $R_{1}, R_{2}, C$ and $L$ along the graphs and $x_{1}(t), x_{2}(t)$ are asymptotically stable as $t \rightarrow \infty$.

In Fig. 5 and Fig. 6, as Assumption (i) and (ii) are satisfied, $x_{1}(t), x_{2}(t)$ are bounded as $t \rightarrow \infty$. The boundedness of $x_{1}(t), x_{2}(t)$ obviously depend on $R_{1}, R_{2}, C$ and $L$. Our result shows that $x_{1}(t), x_{2}(t)$ are bounded by a single constant if the Assumption (i) and (ii) are satisfied but the physical effect on the external conductive elements or connections is not considered.

\section{Conclusion}

Analysis of electrical circuits literary shows that Lyapunov's theory is rarely scarce. The Lyapunov's theory allows us to predict the behavior of control variables characterizing the system of RLC circuit and its response as well as eliminate the undesirable dissipation of such system circuit due to external conductive elements by relatively simple mathematical approach. We have shown how the instability problem in RLC circuit in Fig. 1 can be eliminated if the Assumptions (i) and (ii) obtained by Lyapunov's second or direct method is implemented at the the development stage. 


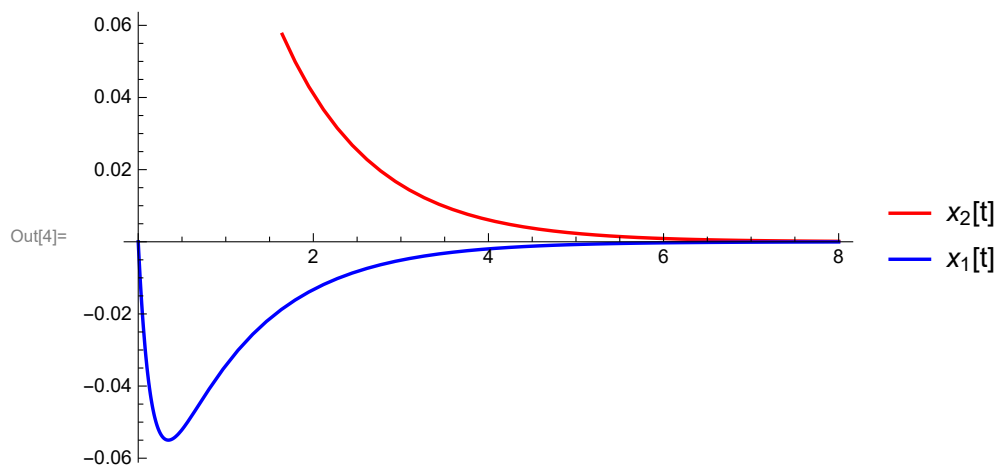

Figure 2: The plot of $x_{1}(t)$ in (blue) and $x_{2}(t)$ in (red) on the same axes

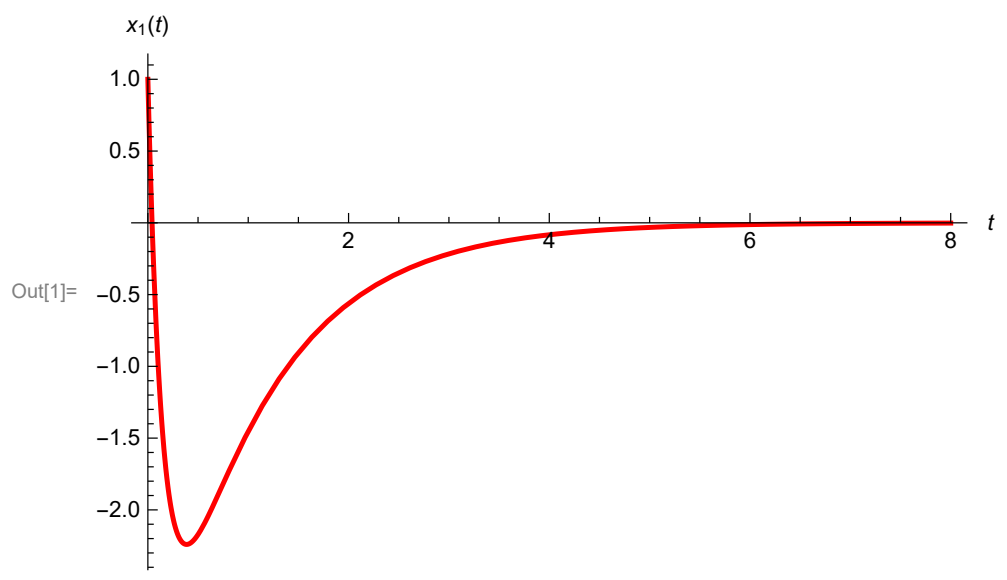

Figure 3: The plot of $x_{1}(t)$ 


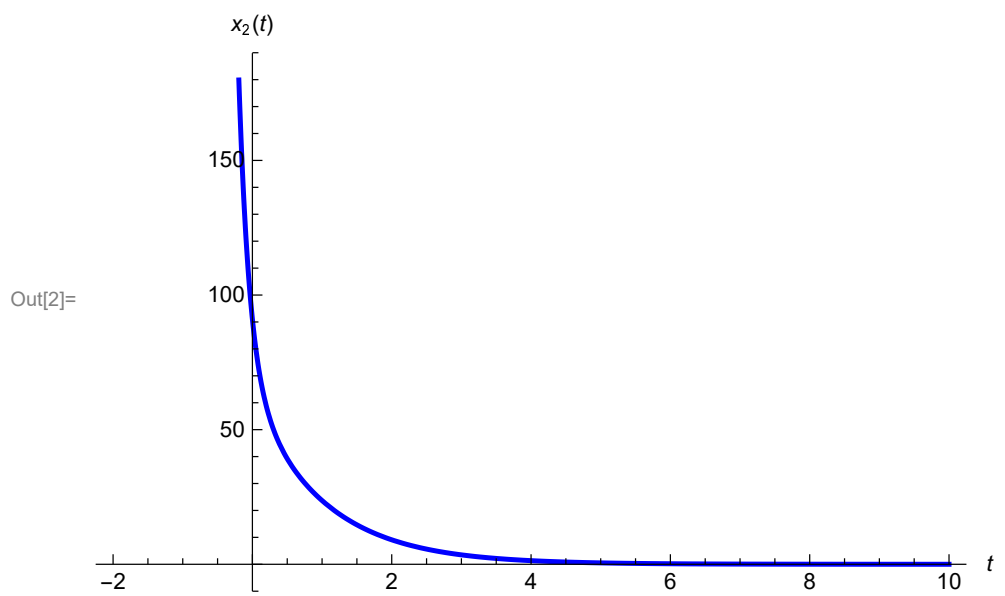

Figure 4: The plot of $x_{2}(t)$

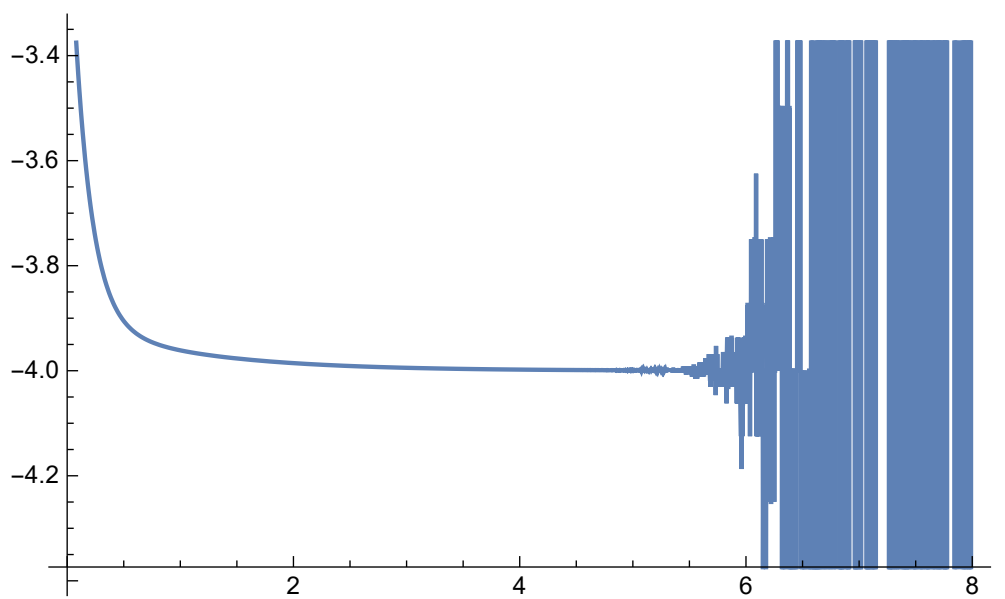

Figure 5: The boundedness of $x_{1}(t)$ 


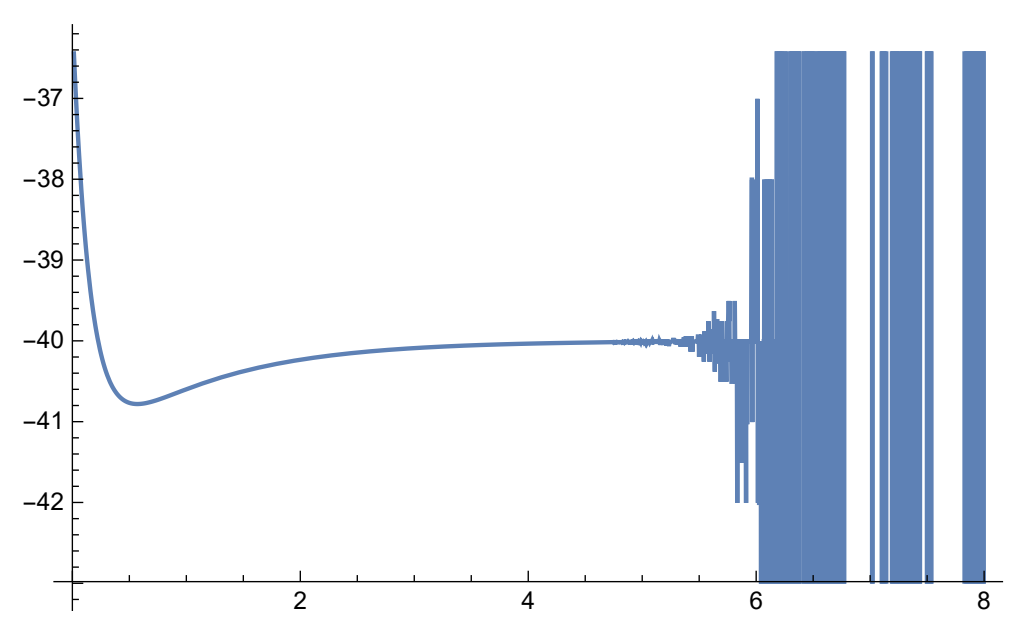

Figure 6: The boundedness of $x_{2}(t)$

\section{References}

[1] B.P. Demidovich, Lectures about Mathematical Stability Theory, Nauka, Moscow (1967).

[2] B.K. Lenka, Time-varying Lyapunov functions and Lyapunov stability of nonautonomous fractional order systems, International Journal of Appled Mathematics, 32, No 1 (2019), 111-130; DOI: 10.12732/ijam.v32i1.11.

[3] A.M. Liapunov, Stability of Motion, Academic Press, London (1966).

[4] N.M. Morris, F.W. Senior, Electric Circuits, Macmillan, Hong Kong (1991).

[5] A.L. Olutimo, Stability analysis of a system of DC servo motor with load, Engineering Math. Letters, 6 (2017), 1-7.

[6] M.A. Pai, Power System stability studies by Lyapunov-Pupov Approach, In: 5th IFAC World Congress, Paris (1972).

[7] D. Piriadarshani, S.S. Sujitha, The role of transfer function in the study of stability analysis of feedback control system with delay, International Journal of Appled Mathematics, 31, No 6 (2018), 727-736; DOI: 10.12732/ijam.v31i6.3.

[8] L. Peng, L.T. Pileggi, NORM: Compact model-order reduction of weakly nonlinear system, In: IEEE/ACM Design Autom. Conf. (2003), 427-477. 
[9] Y. Qin, Y. Liu, L. Wang, Stability of Motion for Dynamic Systems with Delay, Academic Press, Beijing (1966).

[10] C. Tunc, Some remarks on the stability and boundedness of solutions of certain differential equations of fourth-order, Comp. Appl. Math., 26, No 1 (2007), 1-17.

[11] H. Yao, W. Meng, On the stability of solutions of certain nonlinear third order delay differential equations, Int. J. Nonlinear Science, 6, No 3 (2008), 230-237.

[12] T. Yoshizawa, Stability Theory by Lyapunov's Second Method, Math. Soc. Japan (1966). 